\title{
Allergic bronchopulmonary aspergillosis in patients with cystic fibrosis and non-cystic fibrosis bronchiectasis
}

Soheila Alyasin ${ }^{1}$, Mozhgan Moghtaderi², Shirin Farjadian³ ${ }^{3}$ Maryam Babaei ${ }^{4}$, Saeed Hosseini Teshnizi ${ }^{5}$

${ }^{1}$ MD, Allergy Research Center, Department of Pediatrics, Shiraz University of Medical Sciences, Shiraz, Iran

${ }^{2}$ MD, Allergy Research Center, Allergy Clinic of Ali-asghar Hospital, Shiraz University of Medical Sciences, Shiraz, Iran

${ }^{3}$ Ph.D., Professor in Immunology, Allergy Research Center, Department of Immunology, Shiraz University of Medical Sciences, Shiraz, Iran

${ }^{4}$ M.Sc. in Microbiology, Allergy Research Center, Shiraz University of Medical Sciences, Shiraz, Iran

${ }^{5} \mathrm{Ph} . \mathrm{D}$. Candidate in Biostatistics, Clinical Research Development Center of Children Hospital, Hormozgan University of Medical Sciences, Bandar Abbas, Iran

Type of article: Original

\begin{abstract}
Background: Aspergillus sensitization (AS) and allergic bronchopulmonary aspergillosis (ABPA) can occur as a cause of permanent lung damage in patients with cystic fibrosis (CF) and non-CF bronchiectasis.

Objective: The aim of this study was to determine the frequency of AS and ABPA in patients with CF and nonCF bronchiectasis in southwestern Iran.

Methods: This cross-sectional study was conducted on 33 patients with $\mathrm{CF}$ and 27 patients with non-CF bronchiectasis from southwestern Iran who were referred to Namazi Hospital affiliated to Shiraz University of Medical Sciences from July 2015 to February 2016. Skin prick test to Aspergillus fumigatus, peripheral blood eosinophil count, total serum IgE, specific IgE and IgG against Aspergillus fumigatus as well as radiologic chest studies were done for each patient. Statistical analysis was done by Mann-Whitney U test, Fisher Exact test, and Kappa weighted in SPSS software version 18. Level of significance was set at $p<0.05$.

Results: Nine patients with CF (27.3\%) and one patient with non-CF bronchiectasis (3.7\%) had positive skin tests to Aspergillus. There was $81.2 \%$ agreement between positive skin test and specific IgE to Aspergillus fumigatus $(\mathrm{p}<0.001)$. Three patients with CF $(9 \%)$ met the diagnostic criteria for ABPA, whereas ABPA was not seen in patients with non-CF bronchiectasis.

Conclusion: ABPA was low in this study, considering more frequency of AS in patients with cystic fibrosis, clinicians should keep in mind the diagnosis of ABPA for those CF patients that do not respond to usual medical therapy and have positive skin tests to Aspergillus allergens.

Keywords: Aspergillus, Allergic bronchopulmonary aspergillosis, Bronchiectasis, Cystic fibrosis, Fungal antibodies
\end{abstract}

\section{Introduction \\ 1.1. Background and study logic}

Patients with cystic fibrosis (CF) are prone to chronic fungi infections due to increased mucus viscosity in the airways (1-3), and Aspergillus fumigatus (AF) is the most common fungi in the sputum of these patients $(4,5)$. Colonization by AF can mainly manifest as transient carrier, Aspergillus sensitization (AS), Aspergillus colonization and allergic bronchopulmonary aspergillosis (ABPA) in these patients (6). Aspergillus sensitization is the indicator of hypersensitivity to AF which is defined by positive immediate skin reaction to Aspergillus antigens or elevated serum IgE level to AF, and is the first step in the pathogenesis of ABPA (7). The pooled prevalence of AS in

\section{Corresponding author:}

Dr. Mozhgan Moghtaderi, Allergy Research Center, Allergy Clinic of Ali-asghar Hospital, Shiraz University of Medical Sciences, Shiraz, Iran. Tel: +98.713647 4298, Fax: +98.7136474298, Email: Moghtadery@sums.ac.ir

Received: October 30, 2016, Accepted: August 26, 2017, Published: January 2018

iThenticate screening: August 14, 2017, English editing: December 07, 2017, Quality control: December 12, 2017

This article has been reviewed / commented by four experts

(C) 2018 The Authors. This is an open access article under the terms of the Creative Commons Attribution-NonCommercialNoDerivs License, which permits use and distribution in any medium, provided the original work is properly cited, the use is non-commercial and no modifications or adaptations are made. 
patients with $\mathrm{CF}$ has been reported as $39.1 \%$ (8). ABPA is an allergic pulmonary manifestation of repeated inhalation of AF which leads to IgG and IgE formation against AF as well as rising of total IgE (9). A systematic review and meta-analysis study showed the prevalence of ABPA with a pooled prevalence of $8.9 \%$ in patients with CF (8). Diagnosis of ABPA in CF patients is challenging because of overlapping criteria with CF. In recent diagnostic criteria for ABPA proposed by the International Society for Human and Animal Mycology (ISHAM), CF was identified as predisposing conditions for ABPA. Two of the three major criteria including positive immediate skin reactivity to AF or elevated specific IgE levels against AF and elevated total serum $\operatorname{IgE}>417 \mathrm{kU} / \mathrm{L}(>1000$ $\mathrm{IU} / \mathrm{mL}$ ), and two of the three minor criteria including serum precipitating antibodies to aspergillus, elevated $\operatorname{IgG}$ against $\mathrm{AF}$ and peripheral blood eosinophilia count $>500$ cells $/ \mu \mathrm{L}$ as well as new or recent abnormalities on chest radiography or chest computed tomography $(\mathrm{CT})$ consistent with ABPA must be present, to consider a CF patient as ABPA (10). ABPA occurs predominantly in patients with asthma and CF while it has been less frequently noted in other lung diseases such as non-CF bronchiectasis (11). Non-CF bronchiectasis is a respiratory disease with irreversible bronchial dilatation and persistent airway inflammation resulted from multiple inflammatory and infectious injuries to the conducting airways $(12,13)$. The best way for the diagnosis of non-CF bronchiectasis is high-resolution computed tomography (HRCT) of the chest (14).

\subsection{Objectives}

Due to under-diagnosis, CF is rare among Asians. Recent epidemiological studies show a severe course of the disease among the Asian population $(15,16)$. Unrecognized or poorly treated ABPA can lead to airway destruction and pulmonary fibrosis in patients with $\mathrm{CF}$ and non-CF bronchiectasis, resulting in significant morbidity and mortality. The present study was carried out in order to determine the frequency of AS and ABPA in individuals with $\mathrm{CF}$ and non-CF bronchiectasis in southwestern Iran.

\section{Material and Methods}

\subsection{Research design}

This cross-sectional study was carried out on all patients with $\mathrm{CF}$ and non-CF bronchiectasis from southwestern Iran who referred to Namazi Hospital affiliated to Shiraz University of Medical Sciences from July 2015 to February 2016.

\subsection{Selection criteria}

Cystic fibrosis was diagnosed based on the typical clinical features of the disease and sweat chloride concentrations more than $60 \mathrm{mEq} / \mathrm{L}$. The diagnosis of non-CF bronchiectasis was made by the clinical features of the lung disease and result of HRCT of the chest. All volunteered patients with $\mathrm{CF}$ and non-CF bronchiectasis were eligible to enroll in the study. Patients were excluded if they had positive dermatographism for doing skin test. The patients were instructed not to take any anti-histamine for 5 days prior to the skin test. The diagnosis of ABPA was considered based on the criteria of the International Society for Human and Animal Mycology (ISHAM) working Group (10).

\subsection{Data collection}

A questionnaire was used to obtain information about age, sex and history of medications in the last 12 months. Genetic analysis of CF patients and the result of previous chest radiography were recorded from their medical charts.

\subsection{Skin prick test}

Skin prick tests were done for all patients with standard commercial extracts of AF, Penicillium and Alternaria (Greer, Lenoir, NC, USA). Histamine $(10 \mathrm{mg} / \mathrm{mL})$ and saline were used as positive and negative controls, respectively. The results of the skin tests were examined after $15 \mathrm{~min}$ and considered positive when the wheal was $\geq 3 \mathrm{~mm}$ greater in diameter than the negative control.

\subsection{Blood eosinophil, total serum IgE level and aspergillus antibodies test}

The peripheral blood eosinophil count was done by standard hematoxylin and eosin staining, and eosinophil count $>$ $500 \mathrm{cell} / \mu \mathrm{L}$ was considered as eosinophilia. Total serum IgE level (Monobind, Lake Forest, CA, USA) and IgG to AF (IBL International, Hamburg, Germany) were determined with an enzyme-linked immunosorbent assay according to the manufacturers' instructions. Serum specific IgE to AF was done by the ImmunoCAP system (Thermo Fisher-Phadia, Uppsala, Sweden). Total IgE $>200 \mathrm{IU} / \mathrm{mL}$, specific IgG $>12 \mathrm{U} / \mathrm{mL}$ and specific $\operatorname{IgE} \geq 0.1$ $\mathrm{kU} / \mathrm{L}$ were considered positive. 


\subsection{Radiological images}

A recent chest X-ray and HRCT of the chest were analyzed to identify opacities by the same radiologist.

\subsection{Statistical analyses}

Data analyses were performed using descriptive statistics (mean \pm standard deviation, frequency). Fisher Exact test was used to analyze the association between sex and age with skin test. Mann-Whitney U test was used to compare total serum IgE in sex and age groups. Kappa weighted was used to find concordance between skin test, total serum IgE and specific IgE. Analyses were conducted using SPSS version 18 (SPSS Inc., Chicago, IL, USA) and statistical significance was set at $\mathrm{p}<0.05$.

\subsection{Research ethics}

This study was approved by the Ethics Committee of Shiraz University of Medical Sciences (EC-P-92-6337). All patients or their parents in the study were completely voluntary and they read and signed the written informed consent forms prior to the study. Each of the participants could be excluded from the study at any time during the program. Also, confidentiality of all participants' information was ensured.

\section{Results}

Thirty-three patients with CF (14 females and 19 males, mean age $7.8 \pm 6.2$ years, age range 1 to 28 years) and 27 patients with non-CF bronchiectasis (14 females and 13 males, mean age $12.7 \pm 4.4$ years, age range 3.5 to 22 years) were included in this study. All patients were from Shiraz, southwestern Iran. The paraclinical data from these patients are summarized in Table 1. Sensitization to AF was more frequent in male than female patients with CF $(p=0.047)$. Sensitization to AF in CF patients aged less than 16 years did not differ significantly from the older patients (Table 2). There was no correlation between total serum IgE level and sex or age in patients with CF (Table $3)$.

Table 1. Paraclinical characteristic of patients with $\mathrm{CF}$ and non-CF bronchiectasis

\begin{tabular}{|l|l|l|}
\hline Characteristic & CF $(\mathrm{n}=33) ; \mathrm{n}(\%)$ & Non-CF bronchiectasis $(\mathrm{n}=27) ; \mathrm{n}(\%)$ \\
\hline Skin test reactivity to Aspergillus & $9(27.2)$ & $1(3.7)$ \\
\hline Elevated total serum IgE level $>200 \mathrm{IU} / \mathrm{mL}$ & $5(15.1)$ & $4(14.8)$ \\
Elevated total serum IgE level $>417 \mathrm{kU} / \mathrm{L}$ & $7(21.2)$ & $1(3.7)$ \\
\hline Elevated specific IgE to Aspergillus & $11(33.3)$ & $1(3.7)$ \\
\hline Elevated specific IgG to Aspergillus & $14(42.4)$ & $15(55.5)$ \\
\hline Eosinophilia $>500$ cells// $\mu \mathrm{L}$ & $6(18.2)$ & 0 \\
\hline Chest X-ray opacities & $11(33.3)$ & $14(51.8)$ \\
\hline Bronchiectasis & $7(21.2)$ & $27(100)$ \\
\hline
\end{tabular}

Table 2. Comparison of sensitization to Aspergillus fumigatus based on sex and age in patients with cystic fibrosis

\begin{tabular}{|l|l|l|l|l|l|}
\hline Characteristics & \multicolumn{3}{|l|}{ Skin test } & Total & \multirow{2}{*}{ Fisher's Exact Test } \\
\cline { 2 - 5 } & & Positive & Negative & & \\
\hline \multirow{2}{*}{ Sex } & Male & $8(42.1)$ & $11(57.9)$ & $19(57.6)$ & 0.047 \\
\cline { 2 - 5 } & Female & $1(7.1)$ & $13(92.9)$ & $14(42.4)$ & \\
\hline \multirow{2}{*}{ Age (year) } & $\leq 16$ & $6(20.7)$ & $23(79.3)$ & $29(87.9)$ & 0.052 \\
\cline { 2 - 5 } & $>16$ & $3(75.0)$ & $1(25.0)$ & $4(12.1)$ & \\
\hline
\end{tabular}

Table 3. Comparison of total serum IgE level based on sex and age in patients with cystic fibrosis

\begin{tabular}{|l|l|l|l|l|l|c|}
\hline Variables & & $\mathrm{n}$ & Median & $\mathrm{IQR}$ & Mann-Whitney U test & p-value \\
\hline \multirow{2}{*}{ Sex } & Male & 19 & 142.80 & 268.9 & 124.500 & 0.756 \\
\cline { 2 - 8 } & Female & 14 & 48.35 & 212.3 & & \\
\hline \multirow{2}{*}{ Age (year) } & $\leq 16$ & 29 & 74.9 & 237.09 & 30.50 & 0.127 \\
\cline { 2 - 5 } & $>16$ & 4 & 322 & 485.37 & & \\
\hline
\end{tabular}

Total serum IgE $>200 \mathrm{IU} / \mathrm{mL}$ showed no discordance with positive skin test to AF. There was a high agreement between positive skin test and specific IgE to AF (kappa $=81.2 \%, \mathrm{p}<0.001$ ). Also, there was a high agreement between total serum IgE $>200 \mathrm{IU} / \mathrm{mL}$ and IgE specific to AF $>0.1 \mathrm{kU}$ in patients with $\mathrm{CF}$ ( $\mathrm{kappa}=84.3 \%, \mathrm{p}<0.001$ ) but no agreement was seen between specific IgE levels and specific IgG levels to AF. In 10 patients with positive skin tests to Aspergillus, 5 patients had positive skin reaction to Penicillium extract and 1 patient had positive skin 
test to Alternaria. Three patients with CF met the diagnostic criteria for ABPA. Chest x-rays of these patients showed opacities especially in the right upper and middle lobe. The characteristics of these patients are summarized in Table 4. There was no case of ABPA among the patients with non-CF bronchiectasis.

Table 4. Characteristics of three patients with cystic fibrosis who had diagnostic criteria for ABPA

\begin{tabular}{|l|l|l|l|}
\hline Characteristics & Patient 1 & Patient 2 & Patient 3 \\
\hline Age (year) & 16 & 12.5 & 28 \\
\hline Sex & Female & Male & Male \\
\hline Positive skin test to Aspergillus & Yes & Yes & Yes \\
\hline Elevated total serum IgE level & Yes & Yes & Yes \\
\hline Elevated specific IgE to Aspergillus & Yes & Yes & Yes \\
\hline Elevated specific IgG to Aspergillus & Yes & Yes & Yes \\
\hline Eosinophilia & Yes & Yes & Yes \\
\hline Central bronchiectasis & No & Yes & Yes \\
\hline CFTR gene mutation & $2183 \mathrm{AA}>\mathrm{G}$ & R1162X & No mutation \\
\hline
\end{tabular}

\section{Discussion}

Human exposure to fungi is near universal and sensitization to fungi induces ABPA in patients with underlying diseases. The results of skin test reactivity to AF were $27.3 \%$ in patients with $\mathrm{CF}$ and $3.7 \%$ in patients with non-CF bronchiectasis, which were lower than that reported by Valletta et al. They found $56 \%$ sensitization to AF in $222 \mathrm{CF}$ patients older than 6 years (17). Geller et al. also found $66 \%$ sensitization to AF in 51 adult patients with CF (18). Similar to our results, Carneiro et al. found $26 \%$ positive reaction to AF in 74 Brazilian patients with CF (19). A recent meta-analysis study reported the prevalence of positive skin test to AF from $20 \%$ to $65 \%$ in patients with $\mathrm{CF}$, with a pooled prevalence of 39.1\% (8). This variation could be explained by the differences in the AF extracts used for skin tests and the methods of skin tests, whether prick or intradermal. Fungi growth is influenced by climate humidity which is in relation to fungi sensitization. The area of our study is hot and dry and is not suitable for fungi to grow. However, there is a report of AF presence in the dust of $86 \%$ of homes in the studied area, which does not include air-borne fungi allergens (20). In our study, the higher sensitization to AF in male patients with CF can be related to high exposure to fungi in men who often go out. There was a high concordance between positive skin test and specific IgE to AF, therefore, a skin test can be reliably used in screening for AS. Similar to our results, Sharma et al. also found $85 \%$ agreement between total serum $\operatorname{IgE}>500 \mathrm{kU}$ and specific $\operatorname{IgE}$ to $\mathrm{AF}>3.5 \mathrm{kU}$ in Indian children with CF (21). In our study, only $9 \%$ of patients with CF had criteria for ABPA. The mean prevalence of ABPA among 12,447 patients with $\mathrm{CF}$ has been reported to be $7.8 \%$, ranging from $2.1 \%$ in Sweden to $13.6 \%$ in Belgium (9). Maturu et al. in a meta-analysis study, observed a varying prevalence of ABPA in patients with CF from $3 \%$ to $25 \%$, with a pooled prevalence of $8.9 \%$ (8). The lack of a uniform diagnostic criterion and standard tests for the diagnosis of ABPA can be considered the main reasons for this variation. There was no case of ABPA in our patients with non-CF bronchiectasis. Although there are a few reports of ABPA in patients with Kartagener syndrome (as one of the causes of non-CF bronchiectasis), ABPA is rare in patients with non-CF bronchiectasis (22). ABPA has been reported to be more prevalent in adult patients with $\mathrm{CF}$ than in children suffering from $\mathrm{CF}$ (8). All three patients with concurrent $\mathrm{CF}$ and ABPA were older than 12 years in our study. Increased exposure to AF over lifetime, more administration of antibiotics and inhaled corticosteroids have been suggested as potential factors in the increase of ABPA in older patients with CF. Although skin reactivity to AF in patients with $\mathrm{CF}$ is in favor of ABPA, more work ups are required for definitive diagnosis of ABPA. Nevertheless, differential diagnosis of ABPA is important because therapeutic management of ABPA and AS is different. AF is the first causative pathogen in ABPA; the disease can be caused by other members of Aspergillus species as well as other fungi (10). We found sensitization to Penicillium in three patients with CF. Elevated total serum IgE $>417 \mathrm{IU} / \mathrm{mL}$ is as an obligatory criterion in the diagnosis and management of ABPA. High titer of total IgE was seen in 7 (21\%) of our CF patients and $1(3.7 \%)$ patient with non-CF bronchiectasis, whereas only 3 of these patients were considered to have ABPA. Serum IgE levels may be increased in many patients with CF and non-CF bronchiectasis without ABPA, because all serum IgE is not directed to Aspergillus (23). It is proposed that serum IgE level $>1000 \mathrm{IU} / \mathrm{mL}$ will be an important predictive factor for the development of ABPA in the future (24). Among twelve patients with elevated levels of Aspergillus-specific IgE, three were diagnosed as having ABPA. Increased Aspergillus-specific IgE levels in serum is also noted in patients with allergic fungal rhinosinusitis caused by Aspergillus (25). It has been suggested that quantitative measurement of Aspergillus-specific IgG can distinguish patients with ABPA among CF patients (26). This study found that the mean Aspergillus-specific IgG concentration in CF patients with ABPA was 147.5 IU/mL, 
compared to $38.4 \mathrm{IU} / \mathrm{mL}$ in $\mathrm{CF}$ patients without ABPA. It is reported that patients with chronic lung disease often have elevated level of Aspergillus-specific IgG (27). In our study, 55\% of the patients with non-CF bronchiectasis had elevated levels of Aspergillus-specific IgG. As one of the minor criteria in diagnosis of ABPA, eosinophilia was observed in all of our patients with ABPA. Bronchiectasis, one of the diagnostic criteria for ABPA, was diagnosed in 7 (21\%) patients with CF but only 2 of them were diagnosed as having ABPA. The prevalence of bronchiectasis is reported as $50-70 \%$ in patients with $\mathrm{CF}(28,29)$. CF patients with $\triangle \mathrm{F} 508$ mutation are reported to be more susceptible to developing ABPA $(30,31)$, while we detected 2183AA $>\mathrm{G}$ and R1162X mutations in two of the three CF patients with ABPA. Therefore, CFTR gene mutation may make CF patients susceptible to ABPA, but high frequency of $\triangle \mathrm{F} 508$ mutation among $\mathrm{CF}$ patients caused a stronger connection between $\Delta \mathrm{F} 508$ mutation and the ABPA. All patients were recruited from one referral center in Shiraz and therefore the sample size of our patients was limited. Multicenter studies could help to determine the exact prevalence of ABPA in patients with CF and nonCF bronchiectasis in our country. Complicated patients with ABPA will present with worsening of respiratory symptoms, and in this study, we could not consider deterioration of clinical symptoms. ABPA should be considered in patients with $\mathrm{CF}$, especially those patients with positive skin tests to Aspergillus allergens.

\section{Conclusions}

The findings of the present study showed that the number of patients with ABPA was low among patients with CF and non-CF bronchiectasis, and indicated more frequency of AS in these patients. Therefore, it is suggested that in patients with $\mathrm{CF}$ and non-CF bronchiectasis that do not respond to usual medical therapy and have positive skin tests to Aspergillus allergens, treatment for ABPA should be considered. Currently, considering the better diagnosis of $\mathrm{CF}$ and non-CF bronchiectasis, more studies with a larger sample size are suggested.

\section{Acknowledgments:}

This work was supported by a grant from Shiraz University of Medical Sciences (Grant No. 92-01-35-6332).

\section{Conflict of Interest:}

There is no conflict of interest to be declared.

\section{Authors' contributions:}

All authors contributed to this project and article equally. All authors read and approved the final manuscript.

\section{References:}

1) Elborn JS. Cystic fibrosis. Lancet. 2016; 388(10059): 2519-31. doi: 10.1016/S0140-6736(16)00576-6.

2) Kuca K, Klimova B, Novotny M, Maresova P. Cystic Fibrosis Revisited - a Review Study. Med Chem. 2017; 13(2): 102-9. doi: 10.2174/1573406412666160608113235.

3) Farjadian S, Moghtaderi M, Kashef S, Alyasin S, Najib K, Saki F. Clinical and genetic features in patients with cystic fibrosis in southwestern Iran. Iran J Pediatr. 2013; 23(2): 212-5. PMID: 23724185, PMCID: PMC3663315.

4) Liu JC, Modha DE, Gaillard EA. What is the clinical significance of filamentous fungi positive sputum cultures in patients with cystic fibrosis? J Cyst Fibros. 2013; 12(3): 187-93. doi: 10.1016/j.jcf.2013.02.003. PMID: 23491855.

5) Saunders RV, Modha DE, Claydon A, Gaillard EA. Chronic Aspergillus fumigatus colonization of the pediatric cystic fibrosis airway is common and may be associated with a more rapid decline in lung function. Med Mycol. 2016; 54(5): 537-43.

6) Jones AM, Horsley A, Denning DW. What is the importance of classifying Aspergillus disease in cystic fibrosis patients? Expert Rev Respir Med. 2014; 8: 389-92. doi: 10.1586/17476348.2014.915751. PMID: 24869560 .

7) Menzies D, Holmes L, McCumesky G, Prys-Picard C, Niven R. Aspergillus sensitization is associated with airflow limitation and bronchiectasis in severe asthma. Allergy. 2011; 66: 679-85. doi: 10.1111/j.13989995.2010.02542.x. PMID: 21261660.

8) Maturu VN, Agarwal R. Prevalence of Aspergillus sensitization and allergic bronchopulmonary aspergillosis in cystic fibrosis: systematic review and meta-analysis. Clin Exp Allergy. 2015; 45(12): 176578. doi: 10.1111/cea.12595. PMID: 26177981.

9) Mastella G, Rainisio M, Harms HK, Hodson ME, Koch C, Navarro J, et al. Allergic bronchopulmonary aspergillosis in cystic fibrosis. A European epidemiological study. Epidemiologic Registry of Cystic Fibrosis. Eur Respir J. 2000; 16(3): 464-71. 
10) Agarwal R, Chakrabarti A, Shah A, Gupta D, Meis JF, Guleria R, et al. Allergic bronchopulmonary aspergillosis: review of literature and proposal of new diagnostic and classification criteria. Clin Exp Allergy. 2013; 43: 850-73. doi: 10.1111/cea.12141.PMID: 23889240.

11) Moss RB. Fungi in cystic fibrosis and non-cystic fibrosis bronchiectasis. Semin Respir Crit Care Med. 2015; 36(2): 207-16. doi: 10.1055/s-0035-1546750. PMID: 25826588.

12) McShane PJ, Naureckas ET, Tino G, Strek ME. Non-cystic fibrosis bronchiectasis. Am J Respir Crit Care Med. 2013; 188(6): 647-56. doi: 10.1164/rccm.201303-0411CI. PMID: 23898922.

13) Altenburg J, Wortel K, van der Werf TS, Boersma WG. Non-cystic fibrosis bronchiectasis: clinical presentation, diagnosis and treatment, illustrated by data from a Dutch Teaching Hospital. Neth J Med. 2015; 73(4): 147-54. PMID: 25968285.

14) Dodd JD, Lavelle LP, Fabre A, Brady D. Imaging in cystic fibrosis and non-cystic fibrosis bronchiectasis. Semin Respir Crit Care Med. 2015; 36(2): 194-206. doi: 10.1055/s-0035-1546749. PMID: 25826587.

15) Singh M, Rebordosa C, Bernholz J, Sharma N. Epidemiology and genetics of cystic fibrosis in Asia: In preparation for the next-generation treatments. Respirology. 2015; 20(8): 1172-81. doi: 10.1111/resp.12656. PMID: 26437683.

16) Jalalirad M, Houshmand M, Mirfakhraie R, Goharbari MH, Mirzajani F. First study of CF mutations in the CFTR gene of Iranian patients: detection of DeltaF508, G542X, W1282X, A120T, R117H, and R347H mutations. J Trop Pediatr. 2004; 50(6): 359-61. doi: 10.1093/tropej/50.6.359. PMID: 15537723.

17) Valletta E, Braggion C, Mastella G. Sensitization to Aspergillus and allergic bronchopulmonary aspergillosis in a cystic fibrosis population. Pediatr Asthma Allergy Immunol. 1993; 7: 43-9. doi: 10.1089/pai.1993.7.43.

18) Geller DE, Kaplowitz H, Light MJ, Colin A. Allergic bronchopulmonary aspergillosis in cystic fibrosis. Reported prevalence, regional distribution, and patient characteristics. Chest. 1999; 116(3): 639-46. PMID: 10492265.

19) Carneiro AC, Lemos AC, Arruda SM, Santana MA. Prevalence of allergic bronchopulmonaryaspergillosis in patients with cystic fibrosis in the state of Bahia, Brazil. J Bras Pneumol. 2008; 34(11): 900-6. PMID: 19099095.

20) Moghtaderi M, Farjadian S, Fereidouni M, Nasiri M, Nejat A. Indoor Dust Allergen Levels in the Homes of Patients with Childhood Asthma: An Experience From Southwestern Iran. Iran J Allergy Asthma Immunol. 2016; 15(2): 132-7. PMID: 27090366.

21) Sharma VK, Raj D, Xess I, Lodha R, Kabra SK. Prevalence and risk factors for allergic bronchopulmonary aspergillosis in Indian children with cystic fibrosis. Indian Pediatr. 2014; 51(4): 295-7. PMID: 24825267.

22) Sehgal IS, Dhooria S, Bal A, Agarwal R. Allergic bronchopulmonary aspergillosis in an adult with Kartagener syndrome. BMJ Case Rep. 2015; 2015. doi: 10.1136/bcr-2015-211493.

23) Greenberger PA. Allergic bronchopulmonary aspergillosis. J Allergy Clin Immunol. 2002; 110(5): 685-92.

24) Stevens DA, Kan VL, Judson MA, Morrison VA, Dummer S, Denning DW, et al. Practice guidelines for diseases caused by Aspergillus. Infectious Diseases Society of America. Clin Infect Dis. 2000; 30(4): 696709.

25) Ryan MW, Marple BF. Allergic fungal rhinosinusitis: diagnosis and management. Curr Opin Otolaryngol Head Neck Surg. 2007; 15(1): 18-22.

26) Barton RC, Hobson RP, Denton M, Peckham D, Brownlee K, Conway S, et al. Serologic diagnosis of allergic bronchopulmonary aspergillosis in patients with cystic fibrosis through the detection of immunoglobulin G to Aspergillus fumigatus. Diagn Microbiol Infect Dis. 2008; 62(3): 287-91.

27) Agarwal R. Allergic bronchopulmonary aspergillosis. Chest. 2009; 135(3): 805-26.

28) Tepper LA, Caudri D, Rovira AP, Tiddens HA, de Bruijne M. The development of bronchiectasis on chest computed tomography in children with cystic fibrosis: can pre-stages be identified? Eur Radiol. 2016; 26(12): 4563-9. doi: 10.1007/s00330-016-4329-z. PMID: 27108295 PMCID: PMC5101271.

29) Stick SM, Brennan S, Murray C, Douglas T, von Ungern-Sternberg BS, Garratt LW, et al. Bronchiectasis in infants and preschool children diagnosed with cystic fibrosis after newborn screening. J Pediatr. 2009; 155(5): 623-8.e1. doi: 10.1016/j.jpeds.2009.05.005. PMID: 19616787.

30) Walicka-Serzysko K, Sands D. The clinical presentations of pulmonary aspergillosis in children with cystic fibrosis - preliminary report. Dev Period Med. 2015; 19(1): 66-79.

31) Hutcheson PS, Rejent AJ, Slavin RG. Variability in parameters of allergic bronchopulmonary aspergillosis in patients with cystic fibrosis. J Allergy Clin Immunol. 1991; 88(3 Pt 1): 390-4. 